\title{
Experimental Chick Colonization by Campylobacter jejuni
}

\author{
Lindsay Davis $^{1}$ and Victor DiRita ${ }^{2}$ \\ ${ }^{1}$ Department of Microbiology and Immunology, University of Michigan-Ann Arbor, \\ Ann Arbor, Michigan \\ ${ }^{2}$ Unit for Laboratory Animal Medicine, University of Michigan-Ann Arbor, Ann Arbor, \\ Michigan
}

\begin{abstract}
Campylobacter jejuni is commonly isolated from animals, including rodents, farm animals, and especially birds. The most common route of infection for humans is through the ingestion of contaminated chicken. Although $C$. jejuni commonly inhabits a number of animals, it does so asymptomatically. Many animals have been explored for use in pathogenic models; however, the most commonly used and established animal model is the chick colonization model described in this unit. Curr. Protoc. Microbiol. 11:8A.3.18A.3.7. (C) 2008 by John Wiley \& Sons, Inc.
\end{abstract}

Keywords: campylobacteriosis $\bullet$ chicken $\bullet$ colonization $\bullet$ cecum

Campylobacter jejuni is one of the leading causes of bacterial gastroenteritis worldwide. It is primarily acquired through ingestion of contaminated poultry products. Recent advances have improved laboratory maintenance and genetic manipulation of $C$. jejuni, outlined in UNITS $8 A .1 \& 8 A .2$, respectively. This unit is dedicated to the understanding of C. jejuni interactions with its natural host, the chicken.

A number of animals have been used to study $C$. jejuni disease, including chickens, hamsters, ferrets, dogs, primates, rabbits, mice, and pigs (Newell, 2001). Mouse models of infection have been explored, resulting in colonization and limited disease manifestations. However, recent work using immunodeficient mice and limited-flora mice has proven promising in the development of a symptomatic mouse model (Chang and Miller, 2006). Oral inoculation of ferret kits with $C$. jejuni results in diarrhea containing blood and mucus, and was used to assess the virulence of $C$. jejuni strains 81-176 and 11168 (Bacon et al., 2000). Although a promising model, high dosage requirement, cost, and a lack of reagents make it undesirable. Orally inoculated C57BL/6 and IL-10-deficient mice can serve as colonization models for $C$. jejuni. Mansfield et al. (2007) demonstrated that C. jejuni 11168 colonizes the gastrointestinal tract of both animals, with limited pathology in IL-10-deficient mice. Although all the suggested animal models have benefits, the chick colonization model remains the current established animal model

C. jejuni commonly colonizes the avian gut in high numbers, asymptomatically. Colonization can be extensive in the ceca, as high as $1 \times 10^{9}$ to $1 \times 10^{10} \mathrm{cfu} / \mathrm{g}$ cecal contents. An inoculum as little as $10 \mathrm{cfu}$ is able to cause colonization (Hendrixson and DiRita, 2004). Inoculation of chicks of various ages produces a similar outcome. The day-old oral chick model has proven successful in identifying bacterial factors that are necessary for chick colonization. The similarities of $C$. jejuni interactions with the chick gut versus mammalian gut are unknown. However, chick colonization is a crucial part of the infectious cycle for $C$. jejuni, making this model even more appealing.

CAUTION: Campylobacter jejuni is a Biosafety Level 2 (BSL-2) pathogen. Follow appropriate guidelines and regulation for the handling of pathogenic microorganisms.

Current Protocols in Microbiology 8A.3.1-8A.3.7, November 2008

Published online November 2008 in Wiley Interscience (www.interscience.wiley.com).

DOI: $10.1002 / 9780471729259 . \mathrm{mc} 08 \mathrm{a} 03 \mathrm{~s} 11$

Copyright $(2) 2008$ John Wiley \& Sons, Inc.
UNIT 8A.3

BASIC

PROTOCOL

Epsilon

Proteobacteria

8A.3.1

Supplement 11 
Proper hand washing is essential, as $C$. jejuni has been shown to cause gastroenteritis at a small dose (10 to 100 organisms). See UNIT 1A.1 and other pertinent resources for more information.

CAUTION: This experiment requires Animal Biosafety Level 2 (ABSL-2) conditions. Follow all appropriate guidelines for the use and handling of infected animals. See UNIT 1A.1 and other pertinent resources (APPENDIX 1B) for more information.

NOTE: All protocols using live animals must first be reviewed and approved by an Institutional Animal Care and Use Committee (IACUC) and must conform to governmental regulations regarding the care and use of laboratory animals.

NOTE: Unless otherwise stated, all bacterial incubations are to be performed at $37^{\circ} \mathrm{C}$, $85 \% \mathrm{~N}_{2}, 10 \% \mathrm{CO}_{2}$, and $5 \% \mathrm{O}_{2}$.

\section{Materials}

Fertilized Leghorn chicken eggs (Charles River Laboratories)

C. jejuni (UNIT 8A.1)

Mueller-Hinton (MH) agar plates (BD Biosciences) containing $10 \mu \mathrm{g} / \mathrm{ml}$ trimethoprim (TMP)

Phosphate-buffered saline (PBS; APPENDIX 2A)

Isoflurane

$100 \%$ ethanol

MH agar plates (BD Biosciences) containing $10 \mu \mathrm{g} / \mathrm{ml} \mathrm{TMP,} \mathrm{with} \mathrm{and} \mathrm{without}$ $100 \mu \mathrm{g} / \mathrm{ml}$ streptomycin and $30 \mu \mathrm{g} / \mathrm{ml}$ cefaparazone

Rocking incubator (Fig. 8A.3.1; G.Q.F. Manufacturing Company Circulated Air Incubator, Model 1502; https://www.gqfmfg.com)

Water pan with sock wick (built into incubator)

Hygrometer (built into incubator)

Cardboard containers for holding eggs in incubator (supplied with the fertile eggs)

Brooding cages (Model LC-1901-5; Research Equipment Company, Bryan, Texas), $90^{\circ}$ to $95^{\circ} \mathrm{C}$

Heat lamps

1-liter Jam-Jar Chick Drinkers (Amazing Animal Accessories, http://www.amazinganimal.co.uk)

Spectrophotometer

1-ml syringe

Ball-point syringe tips (Fisher Scientific, cat. no. 01-290-4B)

Tight-sealing container (such as a 2- to 3-liter Tupperware container)

Dissecting tools (scalpel, scissors, tweezers)

Petri plates, sterile

Additional reagents and equipment for growing $C$. jejuni under microaerophilic conditions (UNIT 8A.1) and quantifying bacteria by optical density (APPENDIX 4A)

NOTE: It is imperative to keep PBS-infected control chicks, as well as chicks with different inocula, separate. $C$. jejuni is able to transfer between chicks very easily. The authors recommend brooding cages with Plexiglas dividers and separate water and food dishes (initial description in Stern et al., 1988).

\section{Incubate eggs}

The eggs are incubated in a rocking incubator for 21 days.

\section{Experimental}

Chick

Colonization

by $C$. jejuni
1. Receive fertilized eggs.
8A.3.2

Supplement 11 
2. Set the air temperature in the incubator to $100^{\circ} \mathrm{F}\left(37.8^{\circ} \mathrm{C}\right)$.

A higher temperature will kill the embryo and a lower temperature will cause chick underdevelopment.

3. Ensure that the relative humidity is $60 \%$ using a water pan with a cotton sock wick leading to a hygrometer (supplied by incubator manufacturer).

The temperature of the wet bulb should be $86^{\circ} \mathrm{F}\left(30^{\circ} \mathrm{C}\right)$, corresponding to a relative humidity of $58 \%$ to $60 \%$. A sponge placed in the water pan can be used to adjust the relative air humidity.

4. Load eggs into incubator trays, placing smaller side of egg down into tray. Stagger placement of eggs (like a checkerboard) to keep proper circulation.

5. Incubate eggs with rotation until day 17 . Check incubator temperature, humidity, and rotation daily. Also ensure that the water pan is filled at all times.

Automatic egg incubators will rock the shelves daily, simulating the hen's natural rotation of the eggs (Fig. 8A.3.1).

6. On day 17, stop rotation of the racks and position trays horizontally. Reposition eggs by removing them from their cardboard container and laying them onto a hatching tray (trays provided with the incubator and on which the cardboard containers holding the eggs are rested; see Fig. 8A.3.1). If desired, place down absorbent paper in order

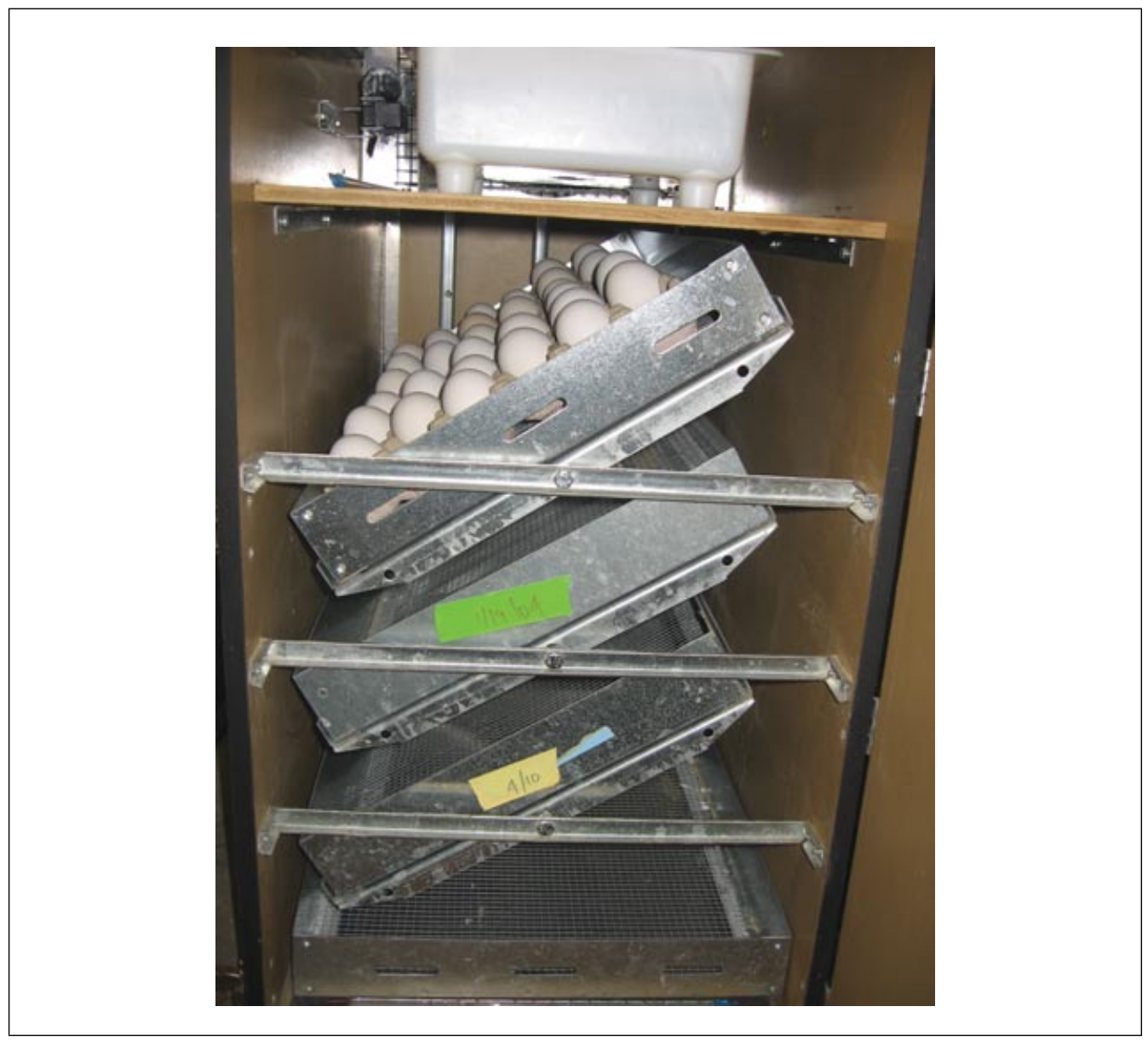

Figure 8A.3.1 Egg placement and interior of rotating incubator is shown here. Eggs are staggered to aid in better air flow between the eggs. A water pan with an absorbent sponge is placed in the incubator to adjust to the desired humidity. 
to help dry the chicks and collect shell debris once they hatch. Continue incubation until eggs hatch (day 21).

At this time, the embryo will position itself for hatching.

IMPORTANT NOTE: Do not adjust position of eggs from this point until hatching, as this will cause the chick to be unable to hatch.

\section{Prepare bacteria}

7. At a time point 3 days prior to hatching, streak out strains from frozen stocks to be investigated (UNIT 8A.1).

This will typically be on day 19.

8. On the day the chicks begin to hatch (day 21) passage the bacteria onto one to two MH plates containing $10 \mu \mathrm{g} / \mathrm{ml}$ TMP (no streptomycin or cefaparazone), depending on the number of chicks being inoculated (typically one plate of bacteria per 10 to 20 chicks). Streak heavily and incubate overnight under microaerophilic conditions (UNIT 8A.1).

\section{Inoculate chicks}

9. One day prior to hatching, set up brooding cages. Place heat lamps over the brooding cages to maintain a temperature between $90^{\circ}$ and $95^{\circ} \mathrm{C}$ (Fig. 8A.3.2).

10. On the day of hatching, allow the chicks 1 day to hatch once they begin breaking the shell.

Helping chicks from shells will slow their progression. Do not help them.

Experimental

Chick

Colonization

by $C$. jejuni

8A.3.4

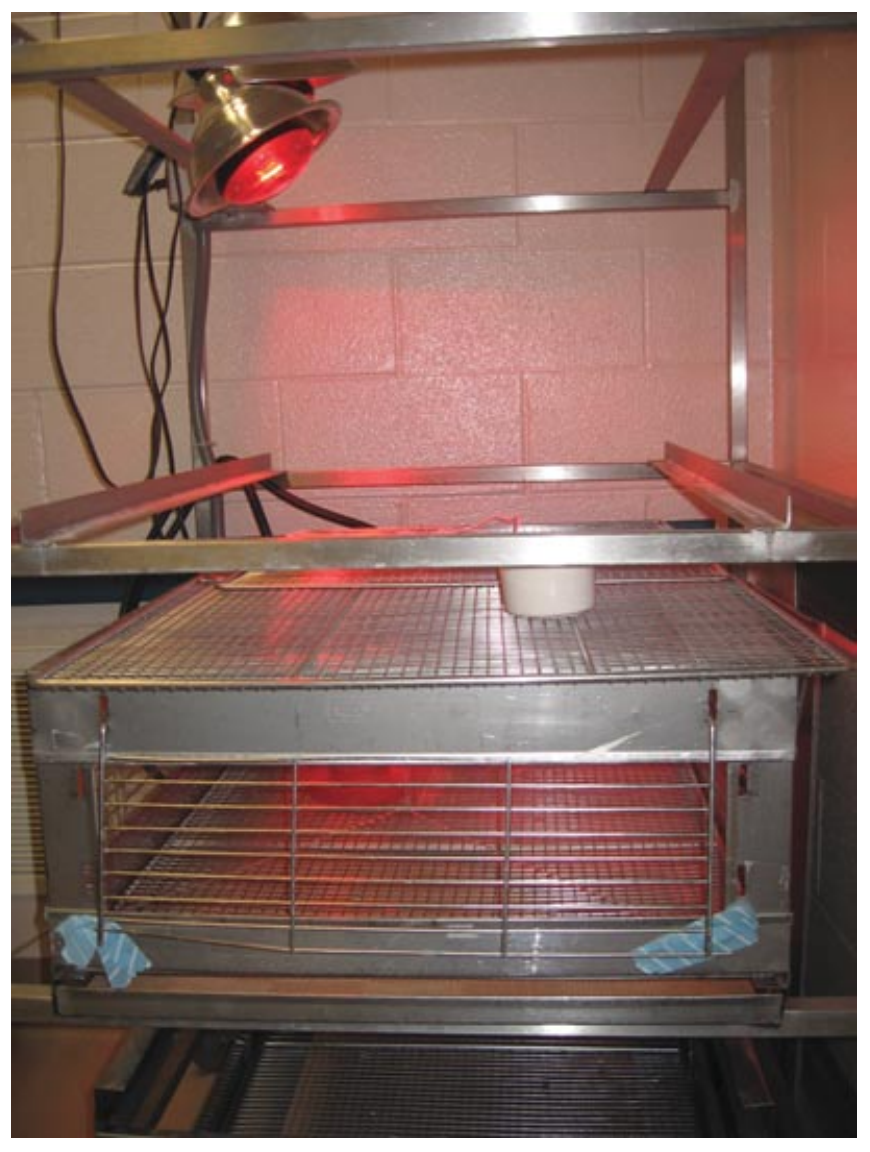

Figure 8A.3.2 Brooding cages with heat lamps are used to house the chicks after hatching. Plexiglas dividers can be used to separate infected chicks. 
11. Once chicks are fully dry and demonstrate the ability to walk, place in brooders. Supply chicks with sterile water immediately in the brooding cage using a plastic 1 Liter Jam-Jar Chick Drinker. Do not feed the chicks until inoculation.

Limiting food for the first day will aid in swallowing of inoculum. Avoid keeping the chicks away from a source of warmth for too long: they are still vulnerable and will begin to shiver.

12. One day post hatching, prepare the inoculum by resuspending the bacteria from 18-hr plates in PBS to an $\mathrm{OD}_{600}$ equal to 0.4 (see APPENDIX 4A).

This equates to $\sim 3$ to $14 \times 10^{8} \mathrm{cfu} / \mathrm{ml}$.

13. Dilute this suspension in PBS to the desired inoculum dosage in a total volume of $100 \mu \mathrm{l}$.

A standard inoculum is $10^{4} \mathrm{cfu} / \mathrm{ml}$.

14. Organize chicks into groups based on whether they are inoculated with PBS alone (control) or $C$. jejuni. If testing several $C$. jejuni strains, keep each group separate from the others. Use a marker on the feathers or toes to distinguish groups.

C. jejuni passes between chicks very easily, so it is imperative to keep them separate.

\section{Infect chicks}

15. Load bacteria into a 1-ml syringe with a ball-point tip.

16. Hold chick in one hand and secure its head with your fingers (Fig. 8A.3.3A).

17. Gently rub the ball-point tip at the corner of the beak until it opens (Fig. 8A.3.3B). Inject $100 \mu \mathrm{l}$ of inoculum. Wait for chirping to ensure inoculum was swallowed.

18. Place chicks in brooding cages in similar groups. Immediately allow access to food and water.

\section{Euthanize chicks}

19. Sacrifice the infected chicks at 7 days post infection by exposure to a lethal dose of anesthesia, preferably isoflurane. For euthanasia by isoflurane, add $1 \mathrm{ml}$ isoflurane to a porous disk in a sealed container. Place the chick in the container and watch for decreased breathing.

Time for euthanasia will be $\sim 5$ min.

Dispose of any unhatched eggs humanely by exposing to high levels of isoflurane in a sealed container. Isoflurane will penetrate the egg shell.

20. Lay chicks on back, exposing abdomen. Pinch skin to ensure no response from the chick.

21. Sterilize the surface of the chick with $100 \%$ ethanol, sprayed across then abdomen.

This will wet the feathers, making the primary incision easier.

22. Sterilize a set of instruments, dissecting scissors, two tweezers, and a scalpel.

Dip the instruments in ethanol and pass through a Bunsen burner.

23. Pinching the skin with tweezers, use the scalpel to make an incision along the abdomen vertically. Make two more incisions perpendicular to the primary incision, forming a $\mathrm{T}$.

24. Using tweezers, spread skin away from abdomen.

25. Expose the intestines by cutting the perineum.

The intestines will spill out.

Epsilon

Proteobacteria

8A.3.5 


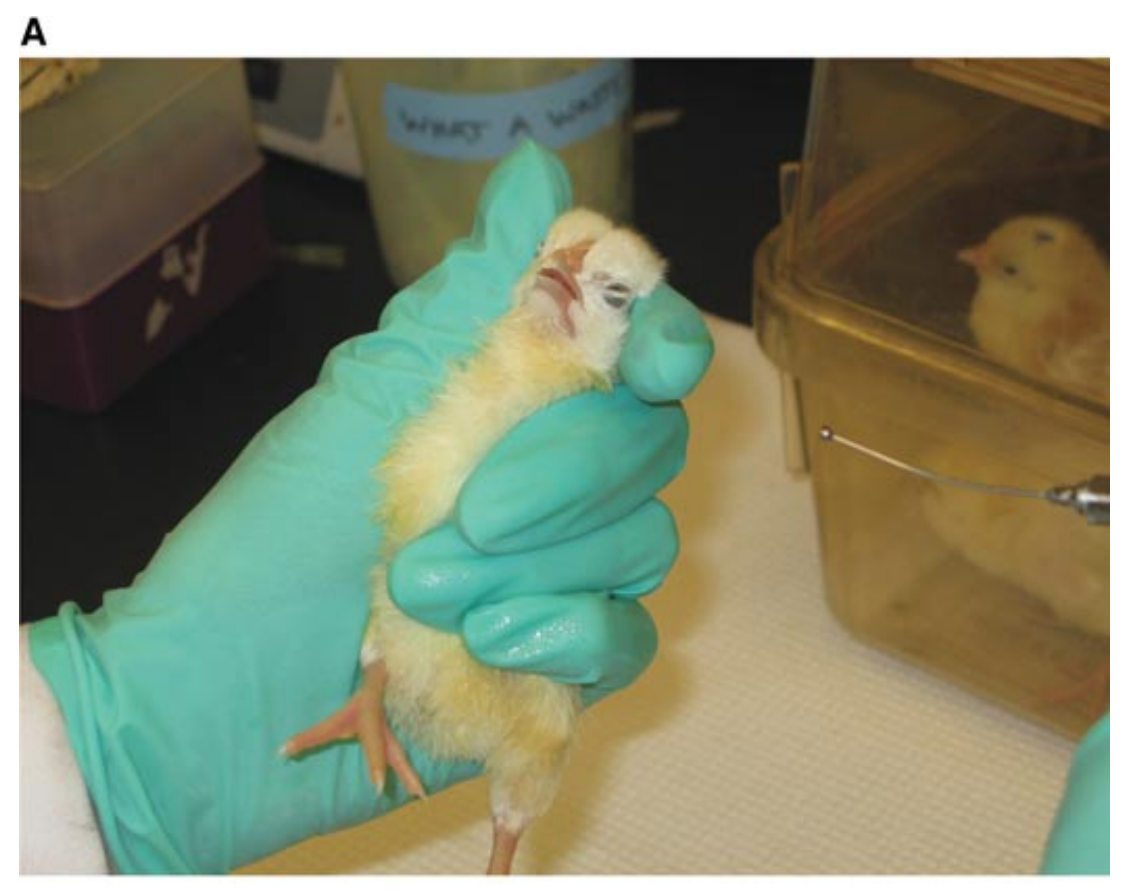

B

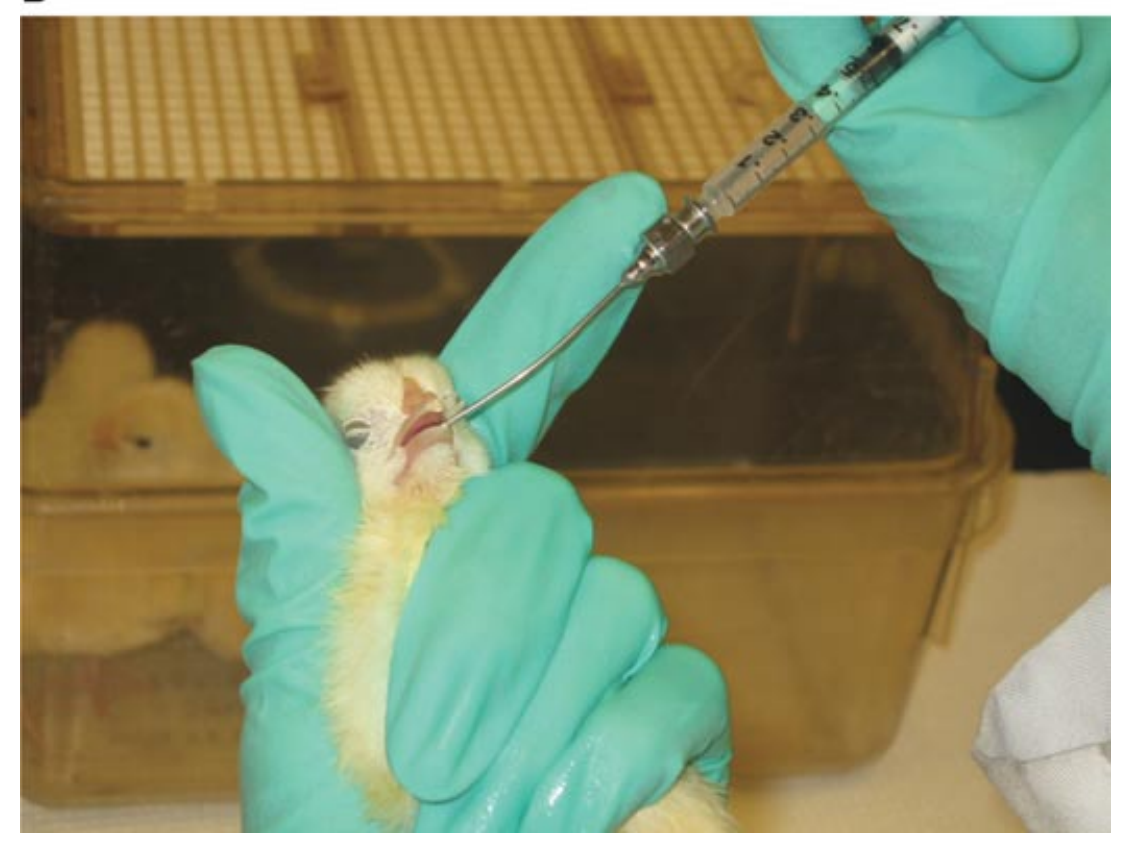

Figure 8A.3.3 (A) To inoculate the chicks, hold the chick in one hand, securing the head with your fingers, as shown. (B) A ball-point tipped syringe is used to deliver the inoculum orally.

26. Find the end of the colon, at the anus, and gently pull the intestines out vertically, working your way up the intestines.

27. Gently remove the ceca from the intestines. Place them in a sterile petri plate. Using a razor or scalpel, cut the distal end of the cecum (this removes the distal muscles, which enables the contents to be squeezed out). Using two sets of tweezers, squeeze the cecal contents into a pre-weighed 1.5 -ml microcentrifuge tube.

The ceca will be two large green-yellow, airy sacks alongside the intestines. 
28. Weigh the tube to get weight of cecal contents and subtract weight of the microcentrifuge tube.

29. Serially dilute cecal contents (ranging from undiluted to $10^{-7}$ ), and plate onto $\mathrm{MH}$ agar plates containing $10 \mu \mathrm{g} / \mathrm{ml} \mathrm{TMP,} 100 \mu \mathrm{g} / \mathrm{ml}$ streptomycin, and $30 \mu \mathrm{g} / \mathrm{ml}$ cefaparazone. Plate undiluted to $10^{-7}$ dilutions.

30. Incubate 2 to 4 days at $37^{\circ} \mathrm{C}$ under microaerophilic conditions (UNIT 8A.1).

\section{COMMENTARY}

\section{Background Information}

Campylobacter jejuni is the causative agent of campylobacteriosis, a self-limiting gastroenteritis. Humans primarily acquire campylobacteriosis through ingestion from contaminated environmental sources such as water, unpasteurized milk, or contaminated chicken products. C. jejuni commonly colonizes the chick gastrointestinal tract, with the cecum being the major organ for colonization.

\section{Critical Parameters and Troubleshooting}

Temperature and humidity are critical for chick development. If underdeveloped chicks hatch (as signified by chicks that have trouble exiting the shell, that have trouble standing hours after hatching, or that are small in size), ensure that the temperature is $100^{\circ} \mathrm{F}\left(37.8^{\circ} \mathrm{C}\right)$ or raise the temperature and humidity slightly. If zero chicks hatch after 22 days, examine an embryo to assess a possible cause. High temperatures will kill the embryo, and repositioning of the egg after day 14 of incubation will cause the chick to be out of position for hatching.

Control inoculations should always be performed to ensure proper inoculation techniques. C. jejuni 81-176 should be used a positive control, with colonization of $10^{9} \mathrm{cfu} / \mathrm{ml}$ being observed 1 to 2 days after inoculation. PBS infection only should result in zero C. jejuni recovery. This control is vital to ensure that cross-contamination between the groups of chicks did not occur. If proper handling and sterile techniques are used, PBS infected chicks can be $C$. jejuni-free throughout the experiment.

\section{Anticipated Results}

The hatch rate of white leghorn eggs is between $60 \%$ and $80 \%$ depending on age of the parenting flock, and incubator specifici- ties. The standard colonization of wild-type C. jejuni $81-176$ is $10^{4} \mathrm{cfu} / \mathrm{ml}$. Nearly $100 \%$ of inoculated animals at this dose become colonized after 7 days.

\section{Time Considerations}

Time considerations for this protocol may vary, depending on variations in the experimental procedure. From start to finish, the outlined chick colonization is completed in 32 days. Chicks hatch within 21 days of fertilization. Oral inoculation occurs at 1 day post hatch with a standard incubation of 1 week. Colonies will appear, if incubated in a tri-gas incubator with selective medium, within 3 to 4 days.

\section{Literature Cited}

Bacon, D.J., Alm, R.A., Burr, D.H., Hu, L., Kopecko, D.J., Ewing, C.P., Trust, T.J., and Guerry, P. 2000. Involvement of a plasmid in virulence of Campylobacter jejuni 81-176. Infect. Immun. 68:4384-4390.

Chang, C. and Miller, J.F. 2006. Campylobacter jejuni colonization of mice with limited enteric flora. Infect. Immun. 74:5261-5271.

Hendrixson, D.R. and DiRita, V.J. 2004. Identification of Campylobacter jejuni genes involved in commensal colonization of the chick gastrointestinal tract. Mol. Microbiol. 52:471-484.

Mansfield, L.S., Bell, J.A., Wilson, D.L., Murphy, A.J., Elsheikha, H.M., Rathinam, V.A., Fierro, B.R., Linz, J.E., and Young, V.B. 2007. C57BL/6 and congenic interleukin-10-deficient mice can serve as models of Campylobacter jejuni colonization and enteritis. Infect. Immun. 75:1099-1115.

Newell, D. G. 2001. Animal models of Campylobacter jejuni colonization and disease and the lessons to be learned from similar Helicobacter pylori models. J. Appl. Microbiol. 90:57S$67 \mathrm{~S}$.

Stern, N.J., Bailey, J.S., Blankenship, L.C., Cox, N.A., and McHan, F. 1988. Colonization characteristics of Campylobacter jejuni in chick ceca. Avian Dis. 32:330-334.
Epsilon

Proteobacteria

8A.3.7

Supplement 11 